\title{
Author Correction: Fit older adults with advanced myelodysplastic syndromes: who is most likely to benefit from transplant?
}

\author{
Gregory A. Abel (1) - Haesook T. Kim - Andrew Hantel (1) - David P. Steensma (1) - Richard Stone - Anand Habib - \\ Vincent T. Ho - Martha Wadleigh - Areej El-Jawahri - Edwin P. Alyea - Daniel J. DeAngelo 1 - John Koreth • \\ Joseph H. Antin (1) - Robert J. Soiffer · Corey Cutler (1)
}

Published online: 24 January 2021

(c) The Author(s), under exclusive licence to Springer Nature Limited 2021

Correction to: Leukemia

https://doi.org/10.1038/s41375-020-01092-2

We have noticed a minor error in "Fig. 3" of the online document, which was introduced during corrections of the proofs when we were asked to add in a description of each panel for the figure.

Everything is correct except in the description/legend at the bottom. To match the label above each panel, the description should have the word "NOT" added into sections $\mathbf{b}$ and d. The legend should read: Fig. 3: Kaplan-Meier Curves for Overall Survival by Entry Criteria and IPSS. a Depicts overall survival for all patients by eligibility criteria (adverse MDS risk versus standard risk with severe cytopenia) and $\mathbf{b}$ for only those NOT undergoing HCT. c Depicts overall survival for all patients by IPSS risk (low or intermediate-1 versus intermediate- 2 or high-risk disease) and $\mathbf{d}$ for only those NOT undergoing HCT. Log-rank testing was used for group comparison.

The original article has been corrected. 